\title{
On the relation between diffusion, sedimentation, and friction
}

\author{
M. M. Kops-Werkhoven and A. Vrij \\ Van't Hoff Laboratorium, Rijks Universiteit Utrecht, Padualaan 8, 3584 CH Utrecht, The Netherlands
}

H. N. W. Lekkerkerker

Faculteit van de Wetenschappen, Vrije Universiteit Brussel, Pleinlaan 2, B 1050 Brussels, Belgium

(Received 14 June 1982; accepted 5 November 1982)

\begin{abstract}
Since there exists in the literature some discrepancies in the formulation of the relation between diffusion, sedimentation, and friction of colloidal particles in solution, we rederive the relation between these transport phenomena in this paper. From the application of irreversible thermodynamics it is shown, that the relation between the experimentally accessible quantities like diffusion coefficient, sedimentation velocity, and osmotic compressibility can be obtained unambiguously. The relation between sedimentation velocity and frictional coefficient is discussed in detail, where we give an argumentation for the right expression between these two quantities.
\end{abstract}

\section{INTRODUCTION}

The relation between the diffusion coefficient, sedimentation velocity and the frictional coefficient for colloidal particles and macromolecules in solution is an old and interesting problem in physical chemistry dating from the time when Nernst, ${ }^{1}$ Einstein $^{2}$ and Svedberg ${ }^{3}$ first gave their fundamental contributions. These relations are important because they allow a consistency check on independent measurements of the above mentioned quantities with techniques such as quasi-elastic light scattering, ultracentrifugation and osmotic methods. In the recent literature, a number of discrepancies are apparent in the formulation of the fundamental relationships involved, with the result that various authors analyze their experimental data in different ways. However, the application of irreversible thermodynamics allows one to establish unambiguously the appropriate relationships as was already shown by Hooyman. ${ }^{4}$

After presenting some historical background on the subject in Sec. II, we indicate the pertinent equations that result from the application of irreversible thermodynamics (Sec. III). In Sec. IV, we discuss the relation between friction and sedimentation in detail. Finally, in Sec. V we denote some sources of error that have led to the confusing situation that exists in the literature.

\section{HISTORICAL BACKGROUND}

A physicochemical description of the process of diffusion of particles in solution was first given about the turn of the century by Nernst, ${ }^{1}$ who recognized that the driving force for the diffusion process is the osmotic pressure gradient in the solution. The elaboration of this approach by Einstein $^{2}$ led to the well-known relation between the diffusion coefficient $D$ of the dispersed particles (component 2) and their frictional resistance, represented by the frictional coefficient $f$. For infinitely diluted systems, (indicated by the subscript 0 ) this relation-nowadays known as the Einstein relation-takes the form

$$
D_{0}=\frac{k_{B} T}{f_{0}},
$$

where $k_{B}$ is the Boltzmann constant and $T$ the absolute temperature. Obviously, the quantities $D$ and $f$ must be taken with respect to the same frame of reference. In this paper, we will further use the volume frame of reference.

With the advent of the ultracentrifuge some 60 years ago, it became feasible to measure the sedimentation velocity $u$ of colloidal particles in solution. At the same time, fundamental theories for the sedimentation velocity were developed. Very familiar are the theoretical considerations of Svedberg, ${ }^{3}$ who, by using the Einstein relation, obtained a relation between the sedimentation constant $s$ and the diffusion coefficient $D$ for infinitely diluted systems.

$$
\frac{s_{0}}{D_{0}}=\frac{M_{2}\left(1-\bar{v}_{2} \rho\right)}{R T},
$$

where $M_{2}$ is the molar mass and $\bar{v}_{2}$ the partial specific volume of the particles, $\rho$ is the density of the solution, which in the limit of infinite dilution is equal to the density of the solvent component $1\left(\rho=\rho_{1}\right)$ and $R$ is the gas constant. The sedimentation constant $s$ is defined by

$$
s=\frac{d x / d t}{\omega^{2} x}=\frac{u}{\omega^{2} x},
$$

where $\omega$ is the angular velocity of the rotation, $x$ is the distance from the rotation axis and $t$ the time.

There is a recent revival of interest in these transport phenomena accompanying the emergence of photon correlation spectroscopy $(\mathrm{PCS})^{5}$ during the last 15 years. With this technique, it became possible to measure the diffusion coefficient of colloidal particles in solution as a function of their concentration, quickly and accurately. In turn, this development became again an incentive for theoretical work on the interrelationships between the experimentally accessible transport quantities, but now with emphasis on their concentration dependence.

However, already in the fifties, Hooyman ${ }^{4}$ showed that, in essence, this problem could be resolved by applying the methods of irreversible thermodynamics. In the next section, we will discuss the results of his deriva- 
tions in relation to the diffusion and sedimentation of colloidal particles.

\section{APPLICATION OF IRREVERSIBLE THERMODYNAMICS}

The application of the theory of irreversible thermodynamics to the required transport phenomena ${ }^{4,6}$ results in a relation between the particle flow $J$ and the force $X$, that is acting on these particles in an (ultra-) centrifuge. In a volume (i.e., laboratory) fixed frame of reference this relation takes the form (contrary to Hooyman ${ }^{4(c)}$ we use the subscript 1 for the solvent and the subscript 2 for the dispersed particles)

$J=L X=L\left[\left(1-\frac{\bar{v}_{2}}{\bar{v}_{1}}\right) \omega^{2} x-\frac{1}{\left(1-\phi_{2}\right)}\left(\frac{\partial \mu_{2}}{\partial c_{2}}\right)_{T, P}\left(\frac{d c_{2}}{d x}\right)\right]$,

where $L$ is the phenomenological constant, $\phi_{2}=\bar{v}_{2} c_{2}$ the volume fraction of the dispersed particles, with $\phi_{1}+\phi_{2}$ $=1, c_{2}$ the mass concentration and $\mu_{2}$ the chemical potential per unit mass of the particles. The derivative of $\mu_{2}$ is taken at constant pressure $P$ and temperature $T$. As can be seen from Eq. (4) the thermodynamic force $X$ consists of two distinct contributions, one due to the centrifugal field and the other due to a concentration gradient in the solution. In the case where only the diffusion process is considered $(\omega=0)$, this equation results in

$$
J=-\frac{L}{\left(1-\phi_{2}\right)}\left(\frac{\partial \mu_{2}}{\partial c_{2}}\right)_{T, P} \frac{d c_{2}}{d x} .
$$

Comparing this result to Fick's law

$$
J=-D \frac{d c_{2}}{d x},
$$

one obtains

$$
D=\frac{L}{\left(1-\phi_{2}\right)}\left(\frac{\partial \mu_{2}}{\partial c_{2}}\right)_{T, P} \quad .
$$

Considering, on the other hand, a sedimentation cell with $\left(d c_{2} / d x\right)=0$, one gets

$$
J=L\left(1-\frac{\bar{v}_{2}}{\bar{v}_{1}}\right) \omega^{2} x .
$$

The sedimentation flow of the colloidal particles can also be formulated by

$$
J=c_{2} u=c_{2} s \omega^{2} x .
$$

From Eqs. (8) and (9), one obtains

$$
s=\frac{L\left[1-\left(\bar{v}_{2} / \bar{v}_{1}\right)\right]}{c_{2}} .
$$

Elimination of the phenomenological coefficient $L$ from Eqs. (7) and (10) results in the following relation between $s$ and $D$ :

$$
\frac{s}{D}=\frac{\left(1-\rho \bar{v}_{2}\right)}{c_{2}\left[\left(\partial \mu_{2} / \partial c_{2}\right)\right]_{T, P}},
$$

where we have used the relation

$$
\left(1-\frac{\bar{v}_{2}}{\bar{v}_{1}}\right)\left(1-\phi_{2}\right)=\left(1-\rho \bar{v}_{2}\right) .
$$

The concentration dependence of the chemical potential $\left(\partial \mu_{2} / \partial c_{2}\right)_{T, P}$ can be related to the osmotic compressi- bility of the system; which is experimentally accessible by light scattering intensity measurements ${ }^{7}$

$$
\left(\frac{\partial \mu_{2}}{\partial c_{2}}\right)_{T, P}=\frac{\left(1-\phi_{2}\right)}{c_{2}}\left(\frac{\partial \Pi}{\partial c_{2}}\right)_{T, \mu_{1}}
$$

(see Appendix), where $\Pi$ is the osmotic pressure and $\mu_{1}$ is the chemical potential of the solvent. Substituting the above equation in Eq. (11) yields

$$
\frac{s}{D}=\frac{\left[1-\left(\bar{v}_{2} / \bar{v}_{1}\right)\right]}{\left(\frac{\partial \Pi}{\partial c_{2}}\right)_{T, \mu_{1}}} .
$$

In the limit of infinite dilution where $\left(\partial \Pi / \partial c_{2}\right)_{T, \mu_{1}}$ $=R T / M_{2}$ and $\rho=\rho_{1}=\bar{v}_{1}^{-1}$, Eq. (14) reduces to Svedberg's Eq. (2).

A useful way to represent the concentration dependence of the experimentally accessible quantities is to write them in the form

$$
\frac{D}{D_{0}}=\frac{s}{s_{0}} \frac{M_{2}}{R T}\left(\frac{\partial \Pi}{\partial c_{2}}\right)_{T, \mu_{1}},
$$

where we have assumed that the partial specific volumes $\bar{v}_{1}$ and $\bar{v}_{2}$ are independent of the particle concentration.

This Eq. (15) reflects the relation between diffusion coefficient, sedimentation constant and osmotic compressibility. In recent work on dispersions of hard spherical silica particles, ${ }^{8}$ this relation has been confirmed experimentally by one of the authors.

\section{RELATION BETWEEN SEDIMENTATION CONSTANT AND FRICTIONAL COEFFICIENT}

The relation between the sedimentation constant and frictional coefficient has been a point of discussion for many years. The problem is centered around the question of how to define the force $F$ that is exerted on a colloidal particle in solution in a centrifugal or gravitational field. In the literature, one can find two different expressions for this force. The first one is, for example, found in the work of Svedberg ${ }^{3}$ and is formulated by

$$
F_{\mathbf{I}}=m_{2}\left(1-\bar{v}_{2} \rho\right) \omega^{2} x,
$$

where $m_{2}$ is the mass per particle, with $m_{2}=M_{2} / N_{\text {Av }}$, $N_{\mathrm{Av}}$ Avogadro's number and $\rho$ is the density of the solution. The other expression for the (ultra) centrifugal force is given by Burgers ${ }^{9}$ :

$$
F_{\mathrm{II}}=m_{2}\left(1-\frac{\bar{v}_{2}}{\bar{v}_{1}}\right) \omega^{2} x=m_{2}\left(1-\bar{v}_{2} \rho_{1}\right) \omega^{2} x,
$$

where $\rho_{1}$ is the density of the solvent. Using Eq. (12), one finds that

$$
F_{\mathrm{I}}=\left(1-\phi_{2}\right) F_{\mathrm{II}} \text {. }
$$

From this relation (18), it follows that the frictional factors $f_{\mathrm{I}}$ and $f_{\mathrm{II}}$, that can be defined by $F=f u$, are related in the same way.

In order to decide which of the two definitions for the centrifugal force is appropriate, we have to examine the situation inside the centrifugal cell in more detail. Here, we follow the reasoning given by Batchelor. ${ }^{10}$ Consider a unit volume of solution consisting of colloidal particles and solvent molecules in the centrifugal cell. 
The centrifugal force is acting on both components in this volume element: $c_{1} \omega^{2} x$ on the solvent, where $c_{1}$ is the mass concentration of the solvent molecules and $c_{2} \omega^{2} x$ on the dispersed particles, while the total force exerted on a unit volume is $\left(c_{1}+c_{2}\right) \omega^{2} x=\rho \omega^{2} x$. However, inside the centrifugal cell, the colloidal particles are moving towards the bottom of the cell, while the solvent molecules change places with these particles (assuming that no volume contraction occurs). When we now consider the force that the colloidal particles undergo while sedimenting, we have to make the solvent molecules forcefree, by exerting an opposite force on this unit volume equal to $c_{1} \omega^{2} x$ divided by $\phi_{1}$. Since this force is also acting on the colloidal particles, the net force per colloidal particle becomes

$F^{*}=\frac{c_{2} \omega^{2} x-\phi_{2}\left[\left(c_{1} \omega^{2} x / \phi_{1}\right)\right]}{n_{2}}=m_{2}\left(1-\frac{\bar{v}_{2}}{\bar{v}_{1}}\right) \omega^{2} x$,

where $n_{2}$ is the number of particles per unit volume of the solution. This equation is equal to the second expression for the force $\left(F_{\text {II }}\right)$ given by Eq. (17).

The result of the above argumentation is that the relation between the sedimentation constant and the frictional coefficient for colloidal particles is

$$
s=\frac{u}{\omega^{2} x}=\frac{F^{*}}{f \omega^{2} x}=\frac{M_{2}\left(1-\bar{v}_{2} \rho_{1}\right)}{N_{\mathrm{Av}} f} .
$$

Inserting this equation in Eq. (14) yields

$$
D=\frac{M_{2}\left[\left(\partial \Pi / \partial c_{2}\right)\right]_{T, \mu_{1}}}{N_{\mathrm{Av}} f} .
$$

This relation (21) can also be obtained by developing the theories of Nernst ${ }^{1}$ and Einstein ${ }^{2}$ to higher particle concentrations, which has been done previously by one of the authors. ${ }^{11}$

\section{DISCUSSION}

From the material presented in the preceding sections, it is clear that-starting from irreversible thermodynamics - the relation between diffusion, sedimentation, and friction can be obtained unambiguously. How ever, this approach has not yet been adopted in a number of well-known and widely applied textbooks. In the older books, like those of Flory ${ }^{12}$ and Tanford, ${ }^{13}$ the relation between $s$ and $D$ is correctly formulated. In some more recent books, there is still no uniformity in the description of the above mentioned phenomena. Actually, a number of discrepancies are apparent and will be discussed here.

In Ref. 5 [Sec. (13.5)], a wrong relation between $\Pi$ and $\mu$ has been used. Equation (13.5.24a) $)^{5}$ is, therefore, incorrect and should be read as

$$
\frac{\partial \ln y_{2}}{\partial c_{2}}=\frac{2 B_{2}}{M_{2}}-\bar{v}_{2},
$$

where $y_{2}$ is the activity coefficient of the particles. However, due to a compensation of errors, the final expression for the diffusion coefficient [Ref. 5 Eq. (13.5.27)] happens to be the right relation.

In Ref. 14 , the term $(-\bar{v})$ in Eq. $(30.45)$ should be omitted. This error can be traced to the use of an in- correct expression of the frictional factor with regard to its definition given in Eq. (30.35). In Ref. 14, Eqs. $(30.35)$ and $(30.36)$ are inconsistent with respect to Eq. (30.34).

In Ref. 15 a factor $\left(1-\phi_{2}\right)$ is erroneously added to the expression for $D$ [Eq. (8.2.35], presumably to account for the frame of reference used. This leads to a relation between $D$ and $s$ [Eq. (8.2.38], which is in error because of this factor.

Finally, the problem discussed here has been extensively treated over the last years by Phillies ${ }^{16}$ who obtains the same result between $D$ and $f$ as we obtained above. He often expresses this result in the form

$$
(D)_{V}=\frac{\left[\left(\partial \Pi / \delta n_{2}\right)\right]\left(1-\phi_{2}\right)}{(f)_{0}},
$$

where $n_{2}$ is the number of particles per unit volume and the subscripts $V$ and 0 refer respectively to the volume and solvent fixed frame of reference. Since

$$
(f)_{0}=(f)_{V}\left(1-\phi_{2}\right) \text {, }
$$

this result is indeed the same as Eq. (21). Note that the frictional coefficient, which can be determined by the ultracentrifuge measurements, is also with regard to the volume fixed frame of reference.

Very recently, a paper by Schurr ${ }^{19}$ appeared discuss ing some aspects of the same problem treated here. The main contention of Schurr is expressed in the first sentence of his summary: "The derivation of mutual translation diffusion coefficients from irreversible thermodynamics is shown to be ambiguous." This is clearly in disagreement with our findings. In the first place, as is obvious from Sec. III of the present paper, the relation between the diffusion coefficient $D$ and the sedimentation constant $s$ can be established unambiguously, leading to the relation (14). Further comparing Eqs. (4) and (19), it follows that the appropriate force to be employed in the definition of the friction factor can be identified without having to invoke any explicit assumptions.

From the discussion above, it appears that errors both from hydrodynamic and thermodynamic origin may occur, while in some publications, the frames of references of the dynamic quantities are not stated explicitly. It is, therefore, more appropriate to compare the measured properties $s, D$, and $\Pi$ directly, as shown in Eqs. (14) or (15).

\section{ACKNOWLEDGMENTS}

The authors are very much indebted to Dr. H. M. Fijnaut for stimulating discussions and valuable criticism in preparing the manuscript. This work is part of the research program of the Foundation for Fundamental Research of Matter (FOM) with financial support from the Netherlands Organization for Pure Research (ZWO).

\section{APPENDIX: DERIVATION ${ }^{17}$ OF EQ. (13)}

Starting from the Gibbs-Duhem relation (at constant temperature):

$$
d P=c_{1} d \mu_{1}+c_{2} d \mu_{2},
$$


it follows that

$$
\left(\frac{\partial P}{\delta c_{2}}\right)_{T, \mu_{1}}=c_{2}\left(\frac{\partial \mu_{2}}{\partial c_{2}}\right)_{T, \mu_{1}} .
$$

The derivative of the chemical potential on the right-hand side of the above equation can be written as $^{18}$

$$
\left(\frac{\partial \mu_{2}}{\partial c_{2}}\right)_{T, \mu_{1}}=\left(\frac{\partial \mu_{2}}{\partial c_{2}}\right)_{T, P}-\frac{\left[\left(\partial \mu_{2} / \partial P\right)\right]_{T, c_{2}}\left[\left(\partial \mu_{1} / \partial c_{2}\right)\right]_{T, P}}{\left[\left(\partial \mu_{1} / \partial P\right)\right]_{T, c_{2}}} .
$$

Using the following relations:

$$
\begin{aligned}
& c_{1}\left(\frac{\partial \mu_{1}}{\partial c_{2}}\right)_{T, P}+c_{2}\left(\frac{\partial \mu_{2}}{\partial c_{2}}\right)_{T, P}=0, \\
& c_{1} \bar{v}_{1}+c_{2} \bar{v}_{2}=1,
\end{aligned}
$$

and

$$
\begin{aligned}
\left(\frac{\partial \mu_{i}}{\partial P}\right)_{T, c_{2}} & =\left(\frac{\partial \mu_{i}}{\partial P}\right)_{T, w}+\left(\frac{\partial \mu_{i}}{\partial w}\right)_{T, P}\left(\frac{\partial w}{\partial P}\right)_{T, c_{2}} \\
& =\bar{v}_{i}-\left(\frac{\partial \mu_{i}}{\partial c_{2}}\right)_{T, P}\left(\frac{\partial c_{2}}{\partial P}\right)_{T, w} \\
& =\bar{v}_{i}-c_{2} \kappa\left(\frac{\partial \mu_{i}}{\partial c_{2}}\right)_{T, P}
\end{aligned}
$$

one finds

$$
\left(\frac{\partial \mu_{2}}{\partial c_{2}}\right)_{T, \mu_{1}}=\frac{\left[1-\left(\kappa / \kappa_{\Pi}\right)\right]\left[\left(\partial \mu_{2} / \partial c_{2}\right)\right]_{T, P}}{c_{1} \bar{v}_{1}}
$$

Here is $w$ the weight fraction

$$
w=c_{2} / \rho,
$$

$\kappa$ the isothermal compressibility

$$
\kappa=-\frac{1}{V}\left(\frac{\partial V}{\partial P}\right)_{T, w}=\frac{1}{\rho}\left(\frac{\partial \rho}{\partial P}\right)_{T, w},
$$

and $\kappa_{\Pi}$ the osmotic compressibility

$$
\kappa_{\mathrm{I}}=\frac{1}{c_{2}\left[\left(\partial P / \partial c_{2}\right)\right]_{T, \mu_{1}}} .
$$

Substitution of Eq. (A7) into (A2) yields

$$
\left(\frac{\partial P}{\partial c_{2}}\right)_{T, \mu_{1}}=\frac{c_{2}\left[1-\left(\kappa / \kappa_{\Pi}\right)\right]}{c_{1} \bar{v}_{1}}\left(\frac{\partial \mu_{2}}{\partial c_{2}}\right)_{T, P} .
$$

For condensed matter $\left(\kappa / \kappa_{\Pi}\right) \ll 1$, that finally reduces Eq. (A11) to Eq. (13):

$$
\left(\frac{\partial P}{\partial c_{2}}\right)_{T, \mu_{1}}=\left(\frac{\partial \Pi}{\partial c_{2}}\right)_{T, \mu_{1}}=\frac{c_{2}}{1-\phi_{2}}\left(\frac{\partial \mu_{2}}{\partial c_{2}}\right)_{T, P} .
$$

${ }^{1}$ W. Nernst, Z. Physik. Chem. 2, 613 (1888).

${ }^{2}$ A. Einstein, Z. Elektrochem. 17, 235 (1908), reprinted in A. Einstein, Investigations on the Theory of the Brownian Movement, edited by R. Fürth (Dover, New York, 1956).

${ }^{3}$ T. Svedberg and K. O. Pederson, Die Ultrazentrifuge (Verlag von Theodor Steinkopf, Dresden/Leipzig, 1940).

${ }^{4}$ (a) G. J. Hooyman, H. J. Holtan, P. Mazur, and S. R. de Groot, Physica 19, 1095 (1953); (b) G. J. Hooyman, ibid. 22, 751, 761 (1956); (c) G. J. Hooyman, Thermodynamics of Diffusion and Sedimentation, Conference on the Ultracentrifuge (Academic, New York, 1963).

${ }^{5}$ B. J. Berne and R. Pecora, Dynamic Light Scattering (Wiley, New York, 1976).

${ }^{6} \mathrm{H}$. Fujita, Foundation of Ultracentrifugal Analysis (Wiley, New York, 1975).

${ }^{7}$ P. J. W. Debye, J. Phys. Chem. 51, 18 (1947).

${ }^{8}$ M. M. Kops-Werkhoven and H. M. Fijnaut, J. Chem. Phys. 74,1618 (1981).

${ }^{9}$ J. M. Burgers, Proc. K. Ned. Akad. Wet. 44, 1177 (1941).

${ }^{10}$ G. K. Batchelor, J. Fluid Mech. 74, 1 (1976).

${ }^{11}$ C. v.d. Broeck, F. Lostak, and H. N. W. Lekkerkerker, J. Chem. Phys. 74, 2006 (1981).

${ }^{12}$ P. J. Flory, Principles of Polymer Chemistry (Cornell University, New York, 1953).

${ }^{13} \mathrm{C}$. Tanford, Physical Chemistry of Marcomolecules (Wiley, New York, 1965).

${ }^{14} \mathrm{H}$. Yamakawa, Modern Theory of Polymer Solutions (Harper and Row, New York, 1971).

${ }^{15}$ B. Chu, Laser Light Scattering (Academic, New York, 1974).

${ }^{16}$ G. D. J. Phillies, J. Chem. Phys. 60, 976, 983 (1974); 62, 3925 (1975); 67, 4690 (1977); 74, 2436 (1981); et al. 75, 508 (1981); J. Colloid Interface Sci. 86, 226 (1982).

${ }^{17}$ A. Vrij, Thesis, Utrecht, 1959, p. 104 :

${ }^{18} \mathrm{E}$. A. Guggenheim, Thermodynamics (North-Holland, Amsterdam, 1974).

${ }^{19}$ J. M. Schurr, Chem. Phys. 65, 217-223 (1982). 